\title{
Single-Unit Activity Patterns in Nuclei That Control the Electromotor Command Nucleus during Spontaneous Electric Signal Production in the Mormyrid Brienomyrus brachyistius
}

\author{
Bruce A. Carlson \\ Department of Neurobiology and Behavior, Cornell University, Ithaca, New York 14853
}

Mormyrid fish generate weak electric organ discharges (EODs) used for communication and navigation. EODs are initiated in the medullary command nucleus $(\mathrm{CN})$, which receives dense projections from the mesencephalic precommand nucleus (PCN) and the adjacent thalamic dorsal posterior nucleus (DP), plus a minor projection from the ventral edge of the toral ventroposterior nucleus (VPv). The dorsal region of the ventroposterior nucleus (VPd) projects to DP-PCN and receives input from the electric organ corollary discharge pathway. I recorded extracellularly from single units within DP-PCN and VPd and correlated their activity patterns with electromotor output to generate hypotheses on electromotor control mechanisms.

DP-PCN neurons show an oscillatory pattern of activity, firing within a window of $10-200$ msec before each EOD, while remaining silent for 50-150 msec after each EOD. VPd neurons only fire during the silent period of DP-PCN neurons, suggesting that they provide recurrent inhibition to DP-PCN. During "scallops", only DP-PCN neurons with high baseline firing rates increase their activity, whereas during "accelerations", only neurons with low baseline firing rates show a strong increase in activity. Thus, the generation of different displays likely results from the activation of different groups of neurons projecting to $\mathrm{CN}$. The activity of VPd neurons decreases during both displays, suggesting that disinhibition plays an important role in their generation. The mormyrid electromotor network shares many functional properties with central pattern generators (CPGs) found in relatively simple motor systems, indicating that it may be an excellent model system for studying CPG function in vertebrate communication.

Key words: motor; command; central pattern generator; corollary discharge; recurrent inhibition; disinhibition

\section{Introduction}

Similar to many behaviors, communication signals are often characterized by stereotyped temporal output patterns orchestrated by central pattern generators (CPGs) (Pollack, 2001). Much of our understanding of the mechanisms controlling the rhythmic output of CPGs comes from studies on relatively simple behaviors such as locomotion, digestion, respiration, and heartbeat (Marder and Bucher, 2001). In this paper, I address electromotor pattern generation in mormyrid fish. Their electric signaling behavior is relatively simple and easily quantified, making them an excellent system for studying the mechanisms underlying rhythmic motor output in vertebrate communication.

Mormyrid electric signals consist of a constant electric organ discharge (EOD) produced with a variable sequence of pulse intervals (SPI) (Hopkins, 1986). The resting SPI has a baseline

Received Aug. 21, 2003; revised Sept. 23, 2003; accepted Sept. 23, 2003.

This work was supported by National Institutes of Mental Health Grant MH37972 and by a National Science Foundation predoctoral fellowship. Special thanks to B. Land and P. Wrege for assistance with data analysis. Previous versions of this manuscript were greatly improved thanks to the suggestions of A. H. Bass, C. D. Hopkins, and J. Sisneros.

Correspondence should be addressed to Bruce A. Carlson, Department of Biology, University of Virginia, Gilmer Hall, P. 0. Box 400328, Charlottesville, VA 22904-4328. E-mail: bc6s@virginia.edu.

Copyright $\odot 2003$ Society for Neuroscience $\quad 0270-6474 / 03 / 2310128-09 \$ 15.00 / 0$ rhythm of EOD intervals ranging from $\sim 100-300 \mathrm{msec}$ (Teyssedre and Boudinot, 1987; Carlson, 2002b). Superimposed on this are a variety of displays, including bursts and cessations, several of which have been linked to specific social contexts (Carlson, 2002b). In Brienomyrus brachyistius, it was recently shown that bursts fall into three categories termed "scallops" (transient, stereotyped bursts; Serrier and Moller, 1989), "accelerations” (graded increases in rate; Carlson, 2002b), and "rasps" (combination of the two; Hopkins and Bass, 1981) based on distinct temporal patterns of EOD production (B. A. Carlson and C. D. Hopkins, unpublished observations).

EODs are initiated in the medullary command nucleus $(\mathrm{CN})$, the output of which is relayed to spinal electromotor neurons (EMN) via the medullary relay nucleus (MRN) (Fig. 1) (Bennett et al., 1967; Bell et al., 1983). CN receives a dense projection from a column of cells within the mesencephalic precommand nucleus $(\mathrm{PCN})$ and the thalamic dorsal posterior nucleus (DP), plus a minor projection from the ventral edge of the toral ventroposterior nucleus (VPv) (Bell et al., 1983; Carlson, 2002a). PCN neurons provide excitatory input to CN (von der Emde et al., 2000); $\mathrm{DP}$ and VPv neurons have not yet been described physiologically.

The medullary electromotor network also gives rise to an ascending corollary discharge pathway that provides an internal 


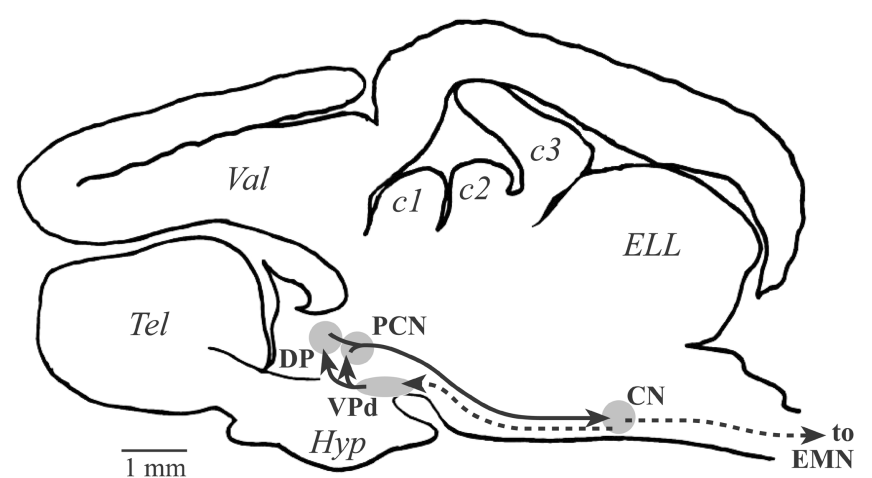

Figure 1. Sagittal schematic of the mormyrid brain, showing the approximate location and extent of CN, PCN, DP, and VPd. Direct connections are indicated by solid lines; indirect connections are indicated by dashed lines. For details on the anatomy, see Bell et al. (1983) and Carlson (2002a).

reference for the timing of EOD output (Bell et al., 1983, 1995). This pathway activates neurons that provide inhibitory input to PCN (von der Emde et al., 2000), thereby establishing a negative feedback loop. It is likely that these inhibitory neurons are located within the dorsal subdivision of the toral ventroposterior nucleus (VPd), which projects to DP-PCN and receives input from the corollary discharge pathway (Carlson, 2002a).

In the current study, I record extracellularly from single units in DP-PCN and VPd to: (1) verify that VPd provides recurrent inhibition to DP-PCN; (2) quantify the diversity of activity patterns among DP-PCN and VPd neurons; (3) analyze the relationship between variation in the resting SPI and single-unit activity; and (4) describe patterns of single-unit activity in relation to the generation of displays. The results suggest several hypotheses on the mechanisms of regulating electromotor output that are likely to be of general relevance in understanding temporal pattern generation in vertebrate communication.

Portions of these results have appeared in abstract form (Carlson and Hopkins, 2000, 2002).

\section{Materials and Methods}

Animals. A total of fifty Brienomyrus brachyistius, ranging in size from 8.0 to $51.0 \mathrm{gm}$ in body weight and from 8.0 to $19.3 \mathrm{~cm}$ in total length were used in this study. Fish were composed of a mix of wild-caught and laboratory-bred fish. They were housed in $280 \mathrm{l}$ group aquaria at a temperature of $25-27^{\circ} \mathrm{C}$ and conductivity of $150-200 \mu \mathrm{S} / \mathrm{cm}$ on a $12 \mathrm{hr}$ light/dark cycle and fed live black worms daily. All procedures were in accordance with the guidelines established by the National Institutes of Health and were approved by the Cornell University Institutional Animal Care and Use Committee.

Surgery. Surgical procedures were identical to those described earlier (Carlson, 2002a). Animals were anesthetized in a solution of $500 \mathrm{mg} / \mathrm{l}$ tricaine methanesulfonate (MS-222; Sigma, St. Louis, MO) and then respirated under a solution of $160 \mathrm{mg} / \mathrm{l}$ MS-222 during the surgery. Fish were placed on a horizontal platform with lateral supports and completely immersed in aquarium water except for the dorsal surface of the head. A flap of skin was removed from the head, and the underlying tissue was scraped away to expose the dorsal surface of the skull. Lidocaine (100-200 $\mu \mathrm{l}$ of a $2 \%$ solution; Radix Laboratories, Inc., Eau Claire, WI) was applied as a local anesthetic. A metal post was affixed to the skull using Superglue (Norpak Adhesives, Lowell, MA) to hold the skull in place, and a small rectangular portion of the skull and meninges were removed to expose the dorsal surface of the midbrain and caudal forebrain. A reference electrode was then placed in the dorsal musculature at the posterior end of the skull. At the end of surgery, the fish were immobilized and electrically silenced with an intramuscular injection of
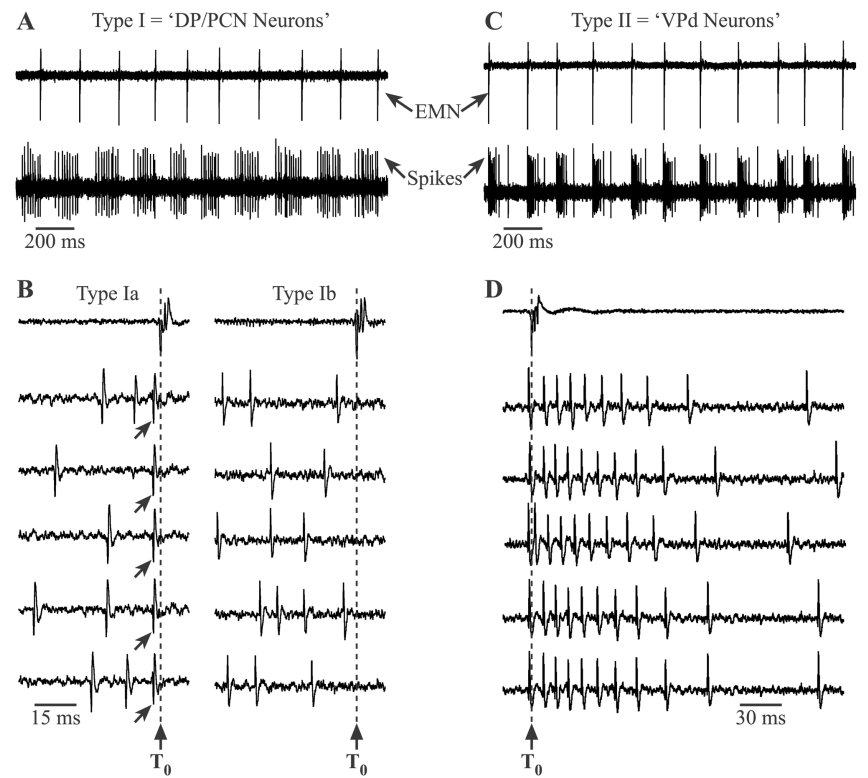

Figure 2. Activity patterns of representative type I units (DP-PCN neurons) and type II units (VPd neurons). A, Continuous recording from a single type I unit. The top trace shows EMN activity, and the bottom trace shows single unit activity. $B$, One example each of type la and type Ib activity patterns. The top traces show a single EMN volley, with a vertical dashed line marking the time of $T_{0}$. The five traces below that show different sweeps from a single unit relative to $T_{0}$. Type la units have an antidromic spike phase-locked to $T_{0}$ (indicated by the upward pointing arrows in each sweep), whereas type lb units do not have phase-locked activity. C, Continuous recording from a single type II unit, constructed as in $A . D$, Expanded view of several of the bursts produced by the type II unit in $C$, constructed as in $B$.

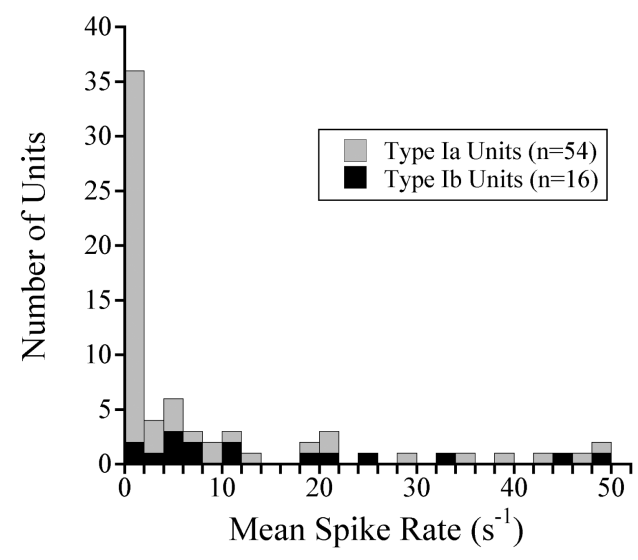

Figure 3. Histogram of mean resting spike rates for all type I units.

flaxedil (gallamine triethiodide: $100-300 \mu \mathrm{l}$ of a $3 \mathrm{mg} / \mathrm{ml}$ solution; Sig$\mathrm{ma})$, and the respiration was switched to fresh water for physiological recording.

Electrophysiology. Electromotor output was monitored by placing a silver wire against the caudal peduncle with a reference several centimeters away. Even though the EOD is silenced by flaxedil, the EOD command can still be recorded as a three-spike potential resulting from the synchronous activation of EMN (Bennett et al., 1967). The first negative peak in the EMN volley was defined as the reference time for EOD output $\left(T_{0}\right)$, which in a natural situation precedes the EOD by $4-5 \mathrm{msec}$. In general, the average EOD (EMN) interval is greater in isolated, curarized preparations than in freely behaving fish, although specific electromotor displays, including scallops and accelerations may still be produced spontaneously.

Glass microelectrodes were pulled using a Sutter Instruments (Novato, CA) Flaming Brown Micropipette Puller, model P-87, and broken to a tip diameter of $<5 \mu \mathrm{m}$. Most electrodes were filled with a solution of 
$3 \mathrm{~m} \mathrm{NaCl}$. In some cases, electrodes were filled with Woods metal and electroplated with gold and platinum (modified from Dowben and Rose, 1953). I localized DP-PCN and VPd first using landmarks on the dorsal surface of the brain and then more precisely by recording characteristic field potentials that were phaselocked to the EMN volley (Carlson, 2002a). Single units were isolated by slowly advancing and retracting the electrode using a microelectrode drive (Inchworm 6000; Burleigh Instruments, Inc., Fishers, NY) that was held by a micromanipulator (model 462-XY-M; Newport Co., Fountain Valley, CA). Extracellular single-unit activity and EMN output were amplified $10,000 \times$ on a differential AC amplifier (model 1700; A-M Systems, Inc., Everett, WA). Singleunit recordings were band-pass filtered from $300-5000 \mathrm{~Hz}$, whereas EMN recordings were band-pass filtered from $10-5000 \mathrm{~Hz}$. Signals were either digitized at $100 \mathrm{kHz}$ on two separate channels of an analog-to-digital board (model AD2; Tucker-Davis Technologies, Gainesville, FL) or sent to two separate Schmitt Triggers with outputs going to event timers that recorded the time of occurrence of spikes and $T_{0}$ using a clock rate of $1 \mathrm{MHz}$ (model ET1; Tucker-Davis Technologies). All data were saved using custom-made software for Matlab 6.5 (The MathWorks, Inc., Natick, MA).

At the end of several experiments $(n=23)$, recording locations were verified by iontophoresis of biotinylated tract-tracers into PCN, $\mathrm{DP}$, or VPd, followed by histological processing (for results, see Carlson, 2002a). In all cases, the injection site was accurately placed using the surface landmarks and field potential characteristics alone, indicating that these methods were sufficient to verify the locations of single-unit recordings in cases in which no injection was made. It was clear that units recorded from at the caudal and rostral ends of DP-PCN were located within PCN and DP, respectively. However, because the two nuclei form a continuous column of cells, it was impossible to determine whether recordings at more intermediate positions were located within PCN or DP. Therefore, all recording locations within these two nuclei are defined as being within DP-PCN rather than precisely within either nucleus.

Data analysis. For analyzing baseline activity levels of single units and their relation to the resting SPI, portions of the records containing burst displays were removed so that only resting output was considered. The relationships between single-unit activity and displays were then analyzed separately. For certain analyses, data on spike times were converted into the spike density function (SDF) through convolution with a Gaussian kernel of a width equal to the mean interspike interval of the unit (Szücs, 1998). The advantages of using the SDF over more traditional means of spike rate analysis have been described elsewhere (Paulin, 1992; Szücs, 1998). Spike timing data and the SPI were analyzed using custommade software for Matlab 6.5. Statistical analyses were done using Statistica 6.1 (StatSoft, Inc., Tulsa, OK) and SAS/STAT 8.02 (SAS Institute, Inc., Cary, NC).

\section{Results}

Two different types of units were encountered while recording within DP-PCN and VPd (Fig. 2). "Type I units" fired sporadically to tonically before $T_{0}$ and then went characteristically silent after $T_{0}$ for tens to hundreds of milliseconds before resuming activity (Fig. $2 A$ ). Within type I units, two distinct subtypes were observed (Fig. 2 B). "Type Ia units" were characterized by single action potentials that were tightly phase-locked with the EMN
Table 1. Quantitative characteristics of the action potential bursts produced by type II units (VPd neurons)

\begin{tabular}{lccl}
\hline Measurement $(n=13)$ & Minimum & Maximum & Mean \pm SEM \\
\hline Timing of first spike relative to $T_{0}$ (msec) & -1.0076 & 7.6942 & $0.5105 \pm 0.6889$ \\
Mean intraburst firing rate $(\mathrm{Hz})$ & 154.09 & 562.05 & $386.41 \pm 35.265$ \\
Mean burst duration (msec) & 5.6519 & 86.933 & $19.944 \pm 6.6834$ \\
Mean number of spikes per burst & 2.7237 & 10.000 & $4.8850 \pm 0.6161$ \\
\hline
\end{tabular}

volley. Across units, the timing of this spike varied from 2.5 to 3.5 msec before $T_{0}$, but it was highly consistent within units, varying in its relative timing by $<100 \mu \mathrm{sec}$. Some type Ia units also had a second phase-locked action potential occurring from $1 \mathrm{msec}$ before to $1 \mathrm{msec}$ after $T_{0}$. By contrast, "type Ib units" did not have any action potentials phase-locked with the EMN volley, although they appeared qualitatively identical in other respects. I recorded from a total of 70 type I units in DP-PCN, of which 54 were type Ia units, and 16 were type Ib units. I did not encounter any type I units in VPd.

"Type II units" were silent throughout the EOD cycle, except for firing a burst of action potentials that started between $\sim 1$ msec before to $8 \mathrm{msec}$ after $T_{0}$, depending on the unit (Fig. 2C,D). I recorded from a total of seven type II units in DP-PCN and six in VPd.

These findings suggest that type I unit recordings were from neurons in DP-PCN, whereas type II unit recordings were from neurons in VPd, as well as the axons of those neurons in DP$\mathrm{PCN}$. This is consistent with a known projection from VPd to DP-PCN (Carlson, 2002a), as well as a previous study on single- 

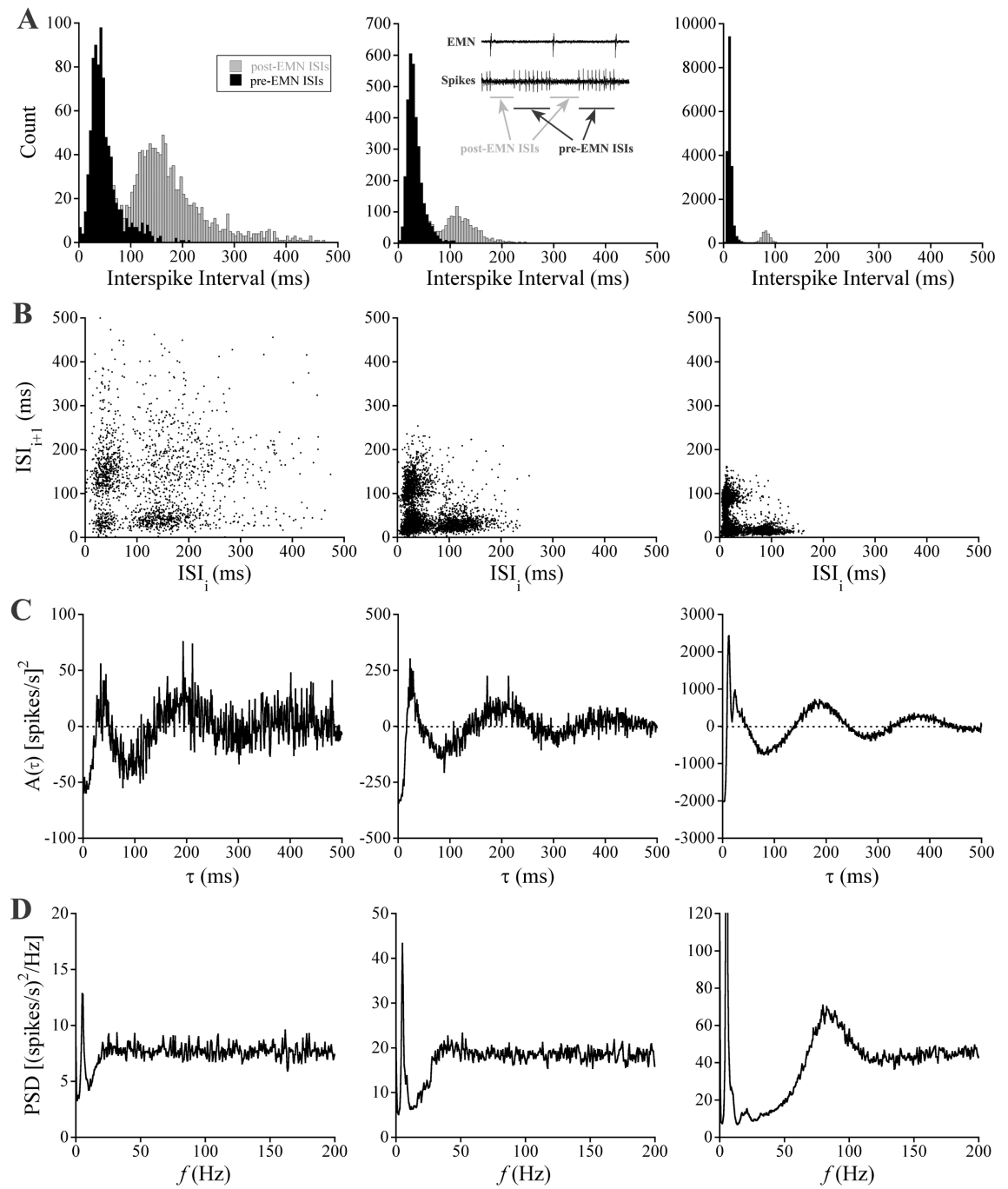

Figure 5. Spike train characteristics of three representative type I units (each with its own column) having different mean spike rates $\left(10.075,21.309\right.$, and $44.777 \mathrm{sec}^{-1}$ from left to right). $A$, ISI histograms. Values are divided into post-EMN ISIs and pre-EMN ISIs, as demonstrated in the inset of the second column. $B$, JHHs show a plot of interspike interval on the abscissa $\left(I S I_{\mathrm{i}}\right)$, against the following interspike interval on the ordinate (ISI $\left.\left.\right|_{i+1}\right)$. C, Autocorrelation functions using a bin size of 1 msec show the relative probability of obtaining a spike at a given delay after a spike at time $0 . D$, Power spectral densities show the relative energy across frequencies for the spike train.

unit physiology in PCN, in which both types of units were encountered (von der Emde et al., 2000). Thus, type I units are defined as DP-PCN neurons, and type II units as VPd neurons.

\section{Variation in single-unit activity}

The phase-locked spikes in type Ia units occur immediately after the generation of an action potential in $\mathrm{CN}$ rather than before, and the available evidence suggests that they result from antidromic activation of axons via electrical synapses in $\mathrm{CN}$ (von der Emde et al., 2000). Because these antidromic spikes are considered a response to $\mathrm{CN}$ activation rather than a cause, the phaselocked spikes were removed in all subsequent analyses of singleunit activity so that they would not artificially increase measures of descending activity in type Ia units.

Both type Ia and Ib units showed wide variation in baseline activity levels from units that fired only sporadically to units that fired at high tonic rates. Across all type I units, the mean spike rate varied from as low as $0 \mathrm{sec}^{-1}$ to as high as $49.24 \mathrm{sec}^{-1}$ (Fig. 3). There was no significant difference in mean spike rate between type Ia and Ib units (Wald-Wolfowitz runs test; $z=1.266 ; p>0.2)$. There did appear to be relatively more type Ia units among those with very low firing rates, although this is likely attributable to a sampling bias because silent type Ib units would have gone unnoticed, whereas silent type Ia units would be identified because of their phase-locked antidromic action potentials.

Looking at mean firing rate in relation to mean EMN interval throughout the entire recording from each unit revealed no significant correlation between mean unit spike rate and mean EMN interval $\left(F_{(1,68)}\right.$ $\left.=0.846 ; r^{2}=0.0123 ; p>0.35\right)$. Thus, neurons with a variety of baseline firing rates were encountered, irrespective of the fish's rate of EOD production.

To analyze the activity patterns of single units in relation to EMN output, rasters and cumulative histograms of spike times relative to $T_{0}$ were constructed (Fig. 4 ). For each type I unit, spike times relative to the previous $T_{0}$ (positive delays) and the following $T_{0}$ (negative delays) were both used (von der Emde et al., 2000). Thus, each spike is shown twice, once as following $T_{0}(i)$ with a given lag and once as preceding $T_{0}(i+1)$ with a given lead. Each type I unit exhibited an increased probability of firing starting from 10 to $150 \mathrm{msec}$ before $T_{0}$ that peaked at 2-6 msec before $T_{0}$ (Fig. $4 A$ ). This peak was very narrow in units with relatively low firing rates (Fig. 4A1,2), whereas it was quite broad in units with higher firing rates (Fig. 4A4-6). Thus, units with high firing rates tended to fire tonically, starting early in the EOD cycle and gradually increasing their activity up to $T_{0}$, whereas units with low firing rates tended to fire only immediately before $T_{0}$. In all units, there was a pronounced decrease in the probability of firing immediately after $T_{0}$ that lasted from 50 to $100 \mathrm{msec}$ (Fig. $4 \mathrm{~A}$ ), resulting from the inhibitory input provided by type II units.

For type II units, each spike was included only once in rasters and histograms as part of a burst centered near $T_{0}$ (von der Emde et al., 2000). Each type II unit was silent throughout the EOD cycle, except for the burst of action potentials, which was more or less stereotyped from cycle to cycle (Fig. $4 B$ ). The first one or two spikes in the burst tended to be especially constant in their presence and timing. There was considerable variation between type II units in the timing of the first spike in the burst relative to $T_{0}$, intraburst firing rate, burst duration, and number of spikes per burst (Table 1).

Interspike interval (ISI) histograms of type I unit activity revealed a characteristic bimodal structure, as is characteristic of oscillatory activity (Fig. 5A). The peak at long intervals resulted from the relatively long pauses after $\mathrm{T}_{0}$ in response to inhibition from type II units, whereas the peak at short intervals resulted from the spikes occurring after these pauses, before the following $T_{0}$. The former are hereafter referred to as post-EMN ISIs, whereas the latter are referred 
to as pre-EMN ISIs (Fig. 5A, inset). When considering units with a mean spike rate of at least $3 \mathrm{sec}^{-1}$, there was a strong correlation between the overall mean pre-EMN ISI and mean post-EMN ISI of a unit $\left(F_{(1,28)}=\right.$ $\left.114.7 ; r^{2}=0.8038 ; p<0.000001\right)$. This relationship did not exist for units with a mean SDF of $<3 \sec ^{-1}\left(F_{(1,25)}=3.225 ; r^{2}=\right.$ $0.1142 ; p>0.08)$, because of their highly infrequent and sporadic activity. Thus, among units that were continuously active, those with high firing rates also tended to resume firing sooner after feedback inhibition.

Resting activity patterns of type I units were also analyzed using joint interval histograms (JIH), autocorrelations, and power spectral densities (Fig. 5B-D) (Gabbiani and Koch, 1998). Like the ISI histograms, all three measures indicate that type I units exhibit properties characteristic of oscillatory activity (Munemori et al., 1984), resulting from a combination of firing at a preferred ISI and periodic interruption of that activity after each $T_{0}$ in response to inhibitory feedback by type II units (Doiron et al., 2003).

\section{Relationships between single-unit activity and the resting SPI}

To analyze the relationship between variation in the activity of individual type I units and the resting SPI, the mean preEMN ISI and the post-EMN ISI before each $T_{0}$ were determined and then compared with the corresponding EMN interval (Fig. 6A). Of 43 total units with a sufficient number of mean pre-EMN ISI values to do a regression analysis, 37 had a significant correlation between mean pre-EMN ISI and EMN interval (Fig. 6Bi,Ci) $\left(r^{2}=0.015-0.613 ; p<0.05\right)$. Of 45 total units with a sufficient number of post-EMN ISI values to do a regression analysis, 41 had a significant correlation between post-EMN ISI and EMN interval (Fig. 6 Bii,Cii) $\left(r^{2}=\right.$ $0.008-0.616 ; p<0.05)$. In an overall analysis of covariance across all units, controlling for unit identity as a repeated measure, there was a highly significant relationship between EMN interval and both mean pre-EMN ISI $\left(F_{(1,20719)}=2909.0 ; p<\right.$ $0.0001)$ and post-EMN ISI $\left(F_{(1,24855)}=2719.56 ; p<0.0001\right)$. When comparing these relationships across units, the slopes of both were positively correlated with mean spike rate (Fig. 6Di) $\left(\right.$ mean pre-EMN ISI; $\left.F_{(1,35)}=18.11 ; r^{2}=0.3410 ; p<0.0002\right)$ (Fig. 6Dii) (post-EMN ISI; $F_{(1,39)}=30.57 ; r^{2}=0.4394 ; p<$ $0.000001)$, indicating that variation in the activity of units with high firing rates has a greater influence on the resting SPI than variation in the activity of units with low firing rates. For mean pre-EMN ISIs, there was a greater increase in the slope with increasing mean spike rate for type Ib units compared with type Ia units (Fig. $6 D i)\left(F_{(1,33)}=5.863 ; p<0.03\right)$, although this was not the case for post-EMN ISIs (Fig. $6 \mathrm{Dii})\left(F_{(1,37)}=1.159 ; p>0.25\right)$.

Although the bursts produced by type II units were fairly stereotyped, there was slight variation within units in the timing of the first spike relative to $T_{0}$, intraburst firing rate, burst duration, and number of spikes per burst. In general however, variation in these variables was not correlated with resting EMN interval within units (Fig. 7). In an overall analysis of covariance across all units, controlling for unit identity as a repeated measure, there was no significant relationship between EMN interval and any of these variables (Bonferoni corrected $p>0.0125$ ). Thus, variation in the resting SPI appears to result primarily from variation in the activity of type I units, but not from variation in the inhibitory feedback provided by type II units.

\section{Relationships between single-unit activity and production of displays}

While recording from type I units, a total of 92 scallops (13 U) and 142 accelerations (30 U) were produced. While recording from type II units, a total of 15 scallops ( $4 \mathrm{U}$ ) and 23 accelerations $(8 \mathrm{U})$ were produced. Rasp production was extremely infrequent, so there were insufficient numbers to analyze neuronal activity during rasps.

Figure 8 shows representative example raster diagrams of the activity of single units during scallop and acceleration production. A type I unit with a relatively high firing rate shows a strong decrease in both pre-EMN ISI and post-EMN ISI during a scallop (Fig. $8 \mathrm{Ai}$ ) and a weaker decrease in pre-EMN ISI and post-EMN ISI during an acceleration (Fig. 8 Aii). By contrast, a type I unit with a relatively low firing rate shows a strong decrease in both pre-EMN ISI and post-EMN ISI during an acceleration (Fig. $8 \mathrm{Bii}$ ), but is fairly silent during a scallop (Fig. $8 \mathrm{Bi}$ ). For type II units, there was a decrease in the number of spikes per burst and 
A

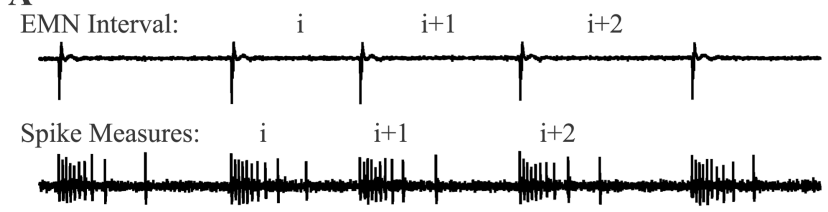

B
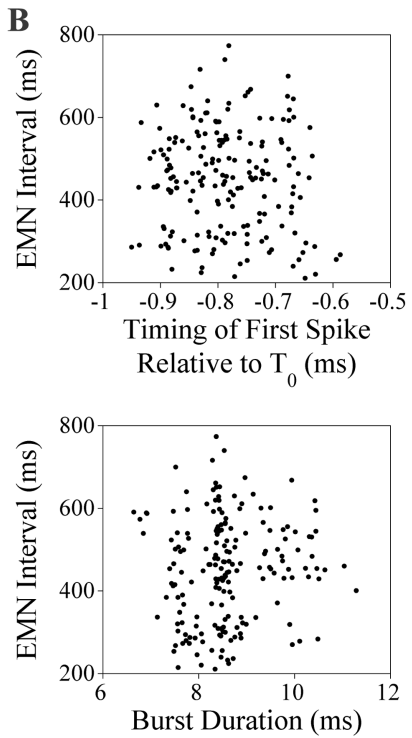
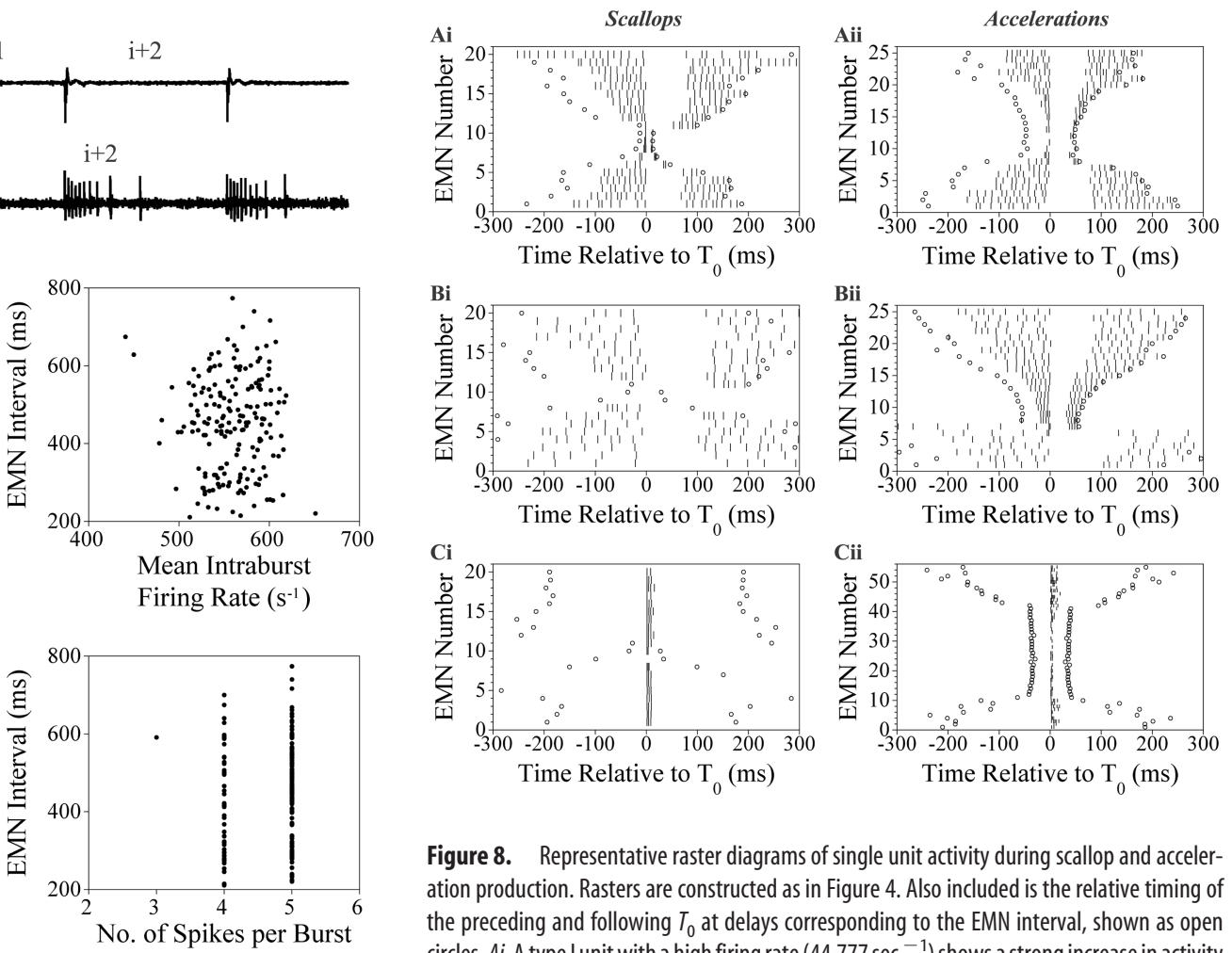

Figure 7. Relationship between the activity of type II units and the resting SPI. $A$, Example showing how four different measures of type II unit activity were correlated with EMN interval within units. $B$, Representative example from a single type II unit of the relationship between resting SPI and these four measures, for which there is no correlation.

burst duration during both scallop (Fig. 8Ci) and acceleration production (Fig. 8Cii).

Figure 9 shows the activity patterns of a single type I unit with a high firing rate during the production of several scallops, along with the single-unit SDF. There is a clear increase in the SDF during the generation of each scallop. However, although the three or four EMNs occurring before the peak (minimum EMN interval) of each scallop are stereotyped in their timing, the temporal pattern of the activity of the unit is quite variable between scallops.

To quantify the patterns of activity across type I and type II units during displays, the peak value of the SDF before each $T_{0}$ during the displays was determined. Because scallops have a stereotyped temporal structure with a distinct peak, it was possible to look at the temporal progression of activity across units relative to scallop production by referencing all activity to the time of occurrence of this peak. Among type I units, those with low baseline firing rates experienced either no change or a decrease in activity during scallop production (Fig. 10A1-8). Only those type I units that fired at high baseline firing rates experienced an increase in the peak SDF during the scallop rise (Fig. 10A9-12). However, depending on the unit, this maximum was reached anywhere from one to four cycles before the scallop peak. Across units, there was an overall significant increase in peak SDF relative to scallop production $\left(F_{(8,632)}=2.019 ; p<0.05\right)$. In addition, there was a significant interaction effect between units and the change in peak SDF relative to scallop production $\left(F_{(96,632)}=\right.$ 4.070; $p<0.000001)$, reflecting the fact that only units with high baseline firing rates showed an increase in activity. Type II units showed a significant decrease in activity during the scallop rise
Bi
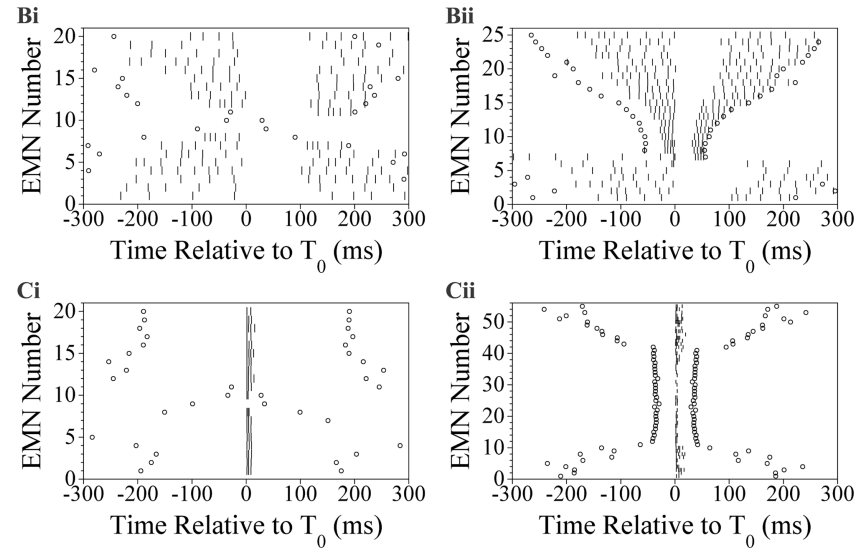

Figure 8. Representative raster diagrams of single unit activity during scallop and acceleration production. Rasters are constructed as in Figure 4. Also included is the relative timing of the preceding and following $T_{0}$ at delays corresponding to the EMN interval, shown as open circles. Ai, A type I unit with a high firing rate ( $\left.44.777 \mathrm{sec}^{-1}\right)$ shows a strong increase in activity during scallop onset, seen as a decrease in pre-EMN ISI and post-EMN ISI. Aii, The same unit as in Ai during an acceleration. There is a decrease in pre-EMN ISI and post-EMN ISI, although it is less dramatic than during the scallop. Bi, A type I unit with a low firing rate (4.7781 $\mathrm{sec}^{-1}$ ) shows no increase in activity during scallop onset and in fact is silent for three cycles near the scallop peak. Bii, The same unit as in Bi during an acceleration. There is a marked decrease in pre-EMN ISI and post-EMN ISI. Ci, A type Il unit experiences a decrease in burst duration and number of spikes per burst during scallop onset. Cii, A second type Il unit shows a similar decrease in activity during an acceleration.

(Fig. $10 B)\left(F_{(8,88)}=3.053 ; p<0.005\right)$. As with type I units, the temporal progression of these changes differed between units, resulting in a significant interaction effect between units and the change in peak SDF relative to scallop production $\left(F_{(24,88)}=\right.$ 1.907; $p<0.02$ ). In one type II unit, there was also a dramatic increase in the peak SDF immediately after the scallop peak (Fig. $10 B 1)$.

Unlike scallops, accelerations are not stereotyped in their temporal structure, so it was not possible to mark a common reference point across accelerations to analyze the temporal progression of activity relative to acceleration production. Thus, changes in activity during accelerations were determined simply by comparing the mean peak SDF during the period before the acceleration to the mean peak SDF during the acceleration. Across type I units, there was a significant increase in the peak SDF during accelerations $\left(F_{(1,112)}=143.5 ; p<0.000001\right)$, although this increase was markedly greater in units with low baseline firing rates (Fig. 11A), leading to a significant interaction effect between units and the change in mean peak $\operatorname{SDF}\left(F_{(29,112)}=5.411 ; p<\right.$ $0.000001)$. Across type II units, there was a significant decrease in the peak SDF during accelerations (Fig. $11 B)\left(F_{(1,15)}=143.5 ; p<\right.$ $0.002)$, although no significant interaction effect between units and the change in mean peak $\operatorname{SDF}\left(F_{(7,15)}=2.243 ; p>0.08\right)$.

\section{Discussion}

The results of the current study identify two types of units in the descending electromotor nuclei of B. brachyistius that are quali- 
A
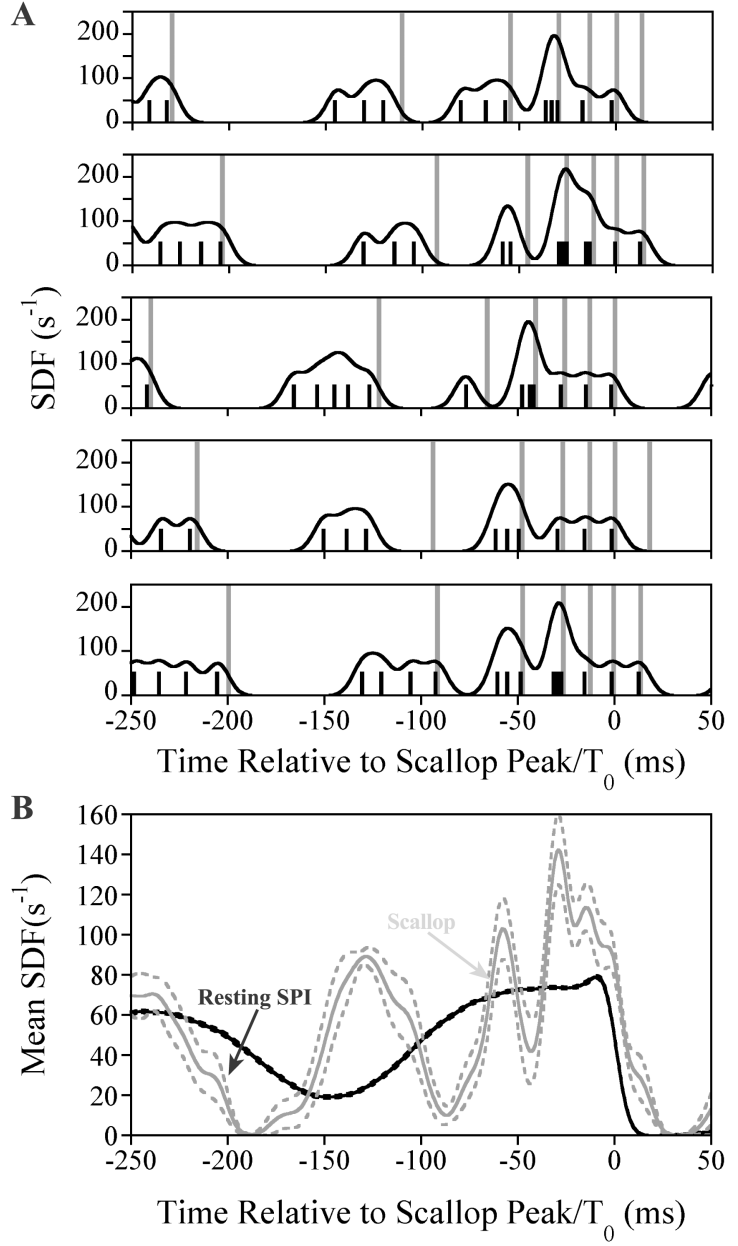

Figure 9. Patterns of activity in a single type I unit with a high baseline firing rate (44.777 $\mathrm{sec}^{-1}$ ) during scallop production. $A$, Five representative examples of activity during scallops. All times are relative to $T_{0}$ at the scallop peak (minimum EMN interval), which is set equal to 0 . The tall, vertical gray lines indicate the relative time of occurrence of each $T_{0}$ during the scallop. The short, vertical black lines indicate the relative time of occurrence of each single-unit spike, and the solid black line indicates the single-unit SDF. B, Average SDF relative to $T_{0}$ during the resting $S P I$ (where 0 is equal to the time of $T_{0}$ ) compared with the average SDF relative to the scallop peaks ( $n=11$ scallops). Solid lines indicate the mean, whereas dashed lines indicate the SEM.

tatively identical to those described in the PCN of Gnathonemus petersii (von der Emde et al., 2000). Type I units (DP-PCN neurons) fire throughout the EOD cycle, except for going silent immediately after each EOD. Type II units (VPd neurons) are silent throughout the EOD cycle, except for producing a stereotyped burst near the time of EOD production, the same time that DPPCN neurons go silent. This finding, combined with an anatomical projection from VPd to DP-PCN and a corollary discharge projection to VPd, strongly suggest that VPd neurons provide recurrent inhibitory input to DP-PCN. An additional group of neurons in VPv project to both DP-PCN and CN, but no recordings have been made in this region, so it remains unclear what role they play in regulating electromotor output.

The available evidence indicates that $\mathrm{CN}$ is not a pacemaker; rather it functions as a command structure that integrates descending activity (Szabo, 1961; Grant et al., 1986, 1999; Carlson, 2002b). Because there is strong electrotonic coupling among CN neurons (Elekes and Szabo, 1985), and they are only active in synchrony (Grant et al., 1986), CN appears functionally equivalent to one large integrate-and-fire neuron, as suggested by a modeling study (Gomez et al., 1998). Descending activity results in a gradual depolarization of $\mathrm{CN}$ until it reaches threshold and generates an EOD (Grant et al., 1986, 1999; Carlson, 2002b).

There was wide variation in the activity patterns of DP-PCN neurons, both in the presence and absence of phase-locked action potentials and in resting rates of activity. Although variation in the activity of DP-PCN neurons was correlated with resting EMN intervals, the strength of this relationship increased with increasing average firing rates. This indicates that units with high baseline firing rates that are continuously active are primarily responsible for the gradual depolarization of $\mathrm{CN}$ neurons that eventually leads to an action potential and EOD generation. However, the infrequent spikes produced by units with lower baseline firing rates tend to occur just before EOD generation and may serve as a final push to drive $\mathrm{CN}$ above threshold. Thus, faster units may widely regulate EOD interval, but slower units may help determine the precise timing of each EOD. Variation in the activity of VPd neurons was not correlated with resting EMN interval, suggesting that they do not contribute to variation in the resting SPI.

During accelerations, DP-PCN neurons with low baseline firing rates experienced a much greater increase in activity than those with high baseline firing rates. By contrast, only those DPPCN neurons with high baseline firing rates experienced an increase in activity during scallops. This suggests that production of the two displays is separated into distinct pathways and results from different patterns of activity across the DP-PCN population. Accelerations appear to result from a recruitment of relatively silent neurons, leading to an increase in the population of units driving $\mathrm{CN}$ and a concomitant increase in the rate and regularity of EOD production, as expected if $\mathrm{CN}$ were serving as an integrate-and-fire unit (Gabbiani and Koch, 1998). During scallops, units that are already firing at high rates and primarily controlling the resting SPI experience a dramatic rise in activity that drives a much more intense, transient burst. Although scallops are highly stereotyped in their temporal structure, the patterns of activity within single units varied dramatically between scallops, indicating that the stereotypy of scallops does not result from stereotyped patterns of input to CN. More likely, this stereotypy results from the biophysical properties of $\mathrm{CN}$ neurons: extremely high rates of activity in a subset of neurons may result in summation, saturation of the $\mathrm{CN}$ membrane potential, and stereotyped output patterns. The available behavioral evidence indicates that rasps result from combining a scallop and acceleration, suggesting that their production may result from combining these two mechanisms.

Two motor control centers for social communication displays In gymnotiform electric fish, which have independently evolved electromotor and electrosensory systems (Bullock et al., 1983), the medullary pacemaker nucleus receives input from two sources analogous to DP and PCN, the central posterior and prepacemaker nuclei, respectively (Carlson, 2002a). The former is responsible for modest, maintained rate increases similar to accelerations, whereas the latter is responsible for intense, transient bursts similar to scallops (Metzner, 1999). Control of scallop and acceleration production may similarly be segregated between PCN and DP, as suggested by preliminary experiments using electrical stimulation (Carlson and Hopkins, 2001). If true, this would suggest a difference in the population of type I units within DP and PCN, with relatively slow units that get recruited during accelerations located in DP and relatively fast units that experience an increase in activity during scallops located in PCN. 
A Type $\mathrm{I}=$ 'DP/PCN Neurons'
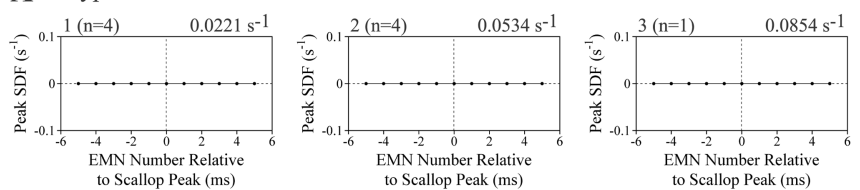
to Scallop Peak (ms)
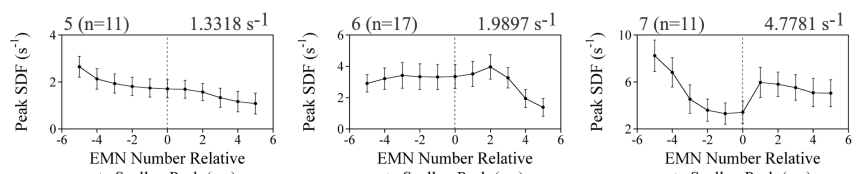
to Scallop Peak (ms)
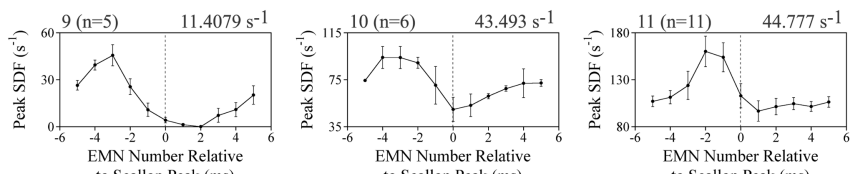
to Scallop Peak (ms)
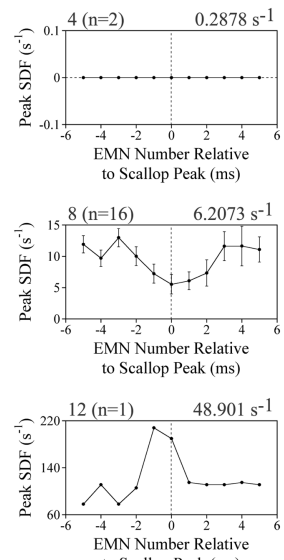

B $\quad$ Type II = 'VPd Neurons'
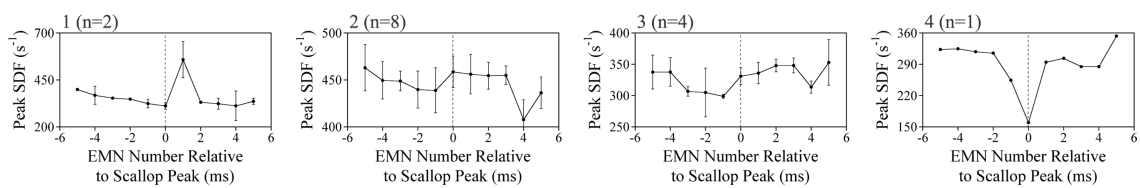

Figure 10. Changes in activity relative to scallop production. $A$, Change in peak SDF during scallop production for 12 of the 13 type I units that were recorded from during scallop production. Units are arranged in order of increasing mean spike rate. The number of scallops produced while recording from that unit is shown in parentheses, and mean spike rate is shown in the top right of each plot. Values shown are the mean peak SDF ( \pm SEM) occurring before each $T_{0}$ during the scallop, with 0 equal to $T_{0}$ at the scallop peak (minimum EMN interval). The one omitted unit had a mean spike rate intermediate to plots 4 and 5 and showed a similar pattern as in plot 4. $B$, Change in peak SDF values during scallop production for the 4 type Il units that were recorded from during scallop production, plotted as in $A$.
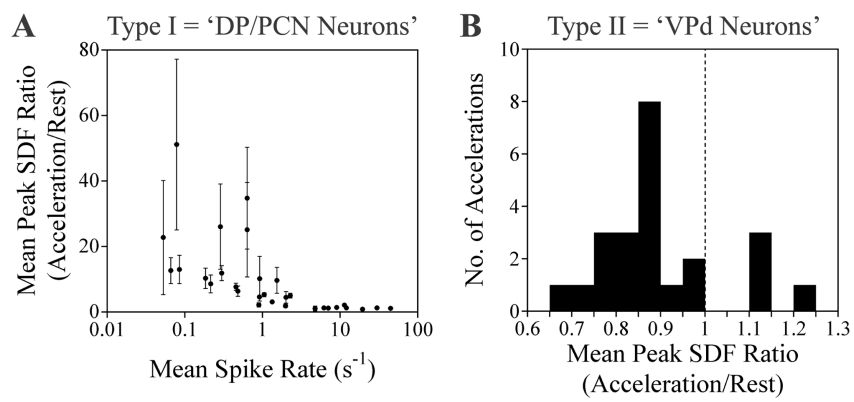

Figure 11. Changes in activity during acceleration production. $A$, Change in mean peak SDF during accelerations for all type I units that were recorded from during acceleration production. Values plotted are the mean ( \pm SEM) of the ratio between the mean peak SDF during each acceleration and the mean peak SDF during the period before each acceleration, plotted against mean spike rate. $B$, Change in mean peak SDF during accelerations for all type II units that were recorded during acceleration production. The histogram shows the ratio between the mean peak SDF during each acceleration and the mean peak SDF during the period before each acceleration. The vertical dashed line indicates a 1:1 ratio (no change in activity).

PCN neurons are larger than DP neurons, and PCN neurons have large extrinsic dendrites, whereas DP neurons have fine intrinsic dendrites (Carlson, 2002a). Such morphological variation is likely related to differences in physiology, further suggesting that variation in activity patterns across type I units may be related to differences between DP and PCN.

The ultrastructure of afferent terminals in CN fall into two categories: club endings with mixed synapses, and bouton-like terminals with chemical synapses (Elekes and Szabo, 1985). Because the phase-locked activity of type Ia units is thought to result from antidromic invasion across electrical synapses (von der to Scallop Peak (ms) to Scallop Peak (ms) to Scallop Peak (ms)

Emde et al., 2000), it is possible that these two morphologies correspond to synapses from type Ia and Ib units, respectively.

\section{Inhibitory mechanisms for behavior}

Variation in activity within VPd neurons did not correlate with variation in the resting SPI. However, their activity was reduced during scallops and accelerations, suggesting that generating these displays results at least in part from a reduction in negative feedback. Disinhibition would free DP-PCN neurons from their ratelimiting factor, allowing them to drive bursts. Similar disinhibitory mechanisms for generating stereotyped motor output have been described in other systems (Noga et al., 1988; Wang and Bieger, 1991; Faumont et al., 1998), and experiments are currently testing this hypothesis. It is unclear what may drive disinhibition, but the tectum mesencephali has a strong projection to VPd that may provide a modulatory input (Wullimann and Northcutt, 1990; Carlson, 2002a). In addition to burst displays, mormyrids may produce cessations of electromotor output (Moller et al., 1989). It is possible that they result from an increase in inhibitory feedback from VPd, thereby removing the excitatory input to $\mathrm{CN}$.

The inhibitory feedback provided by VPd may also be involved in establishing physiological differences among DP-PCN neurons. There was wide variation in activity among VPd neurons, and this variation could drive differences in the baseline activity levels of DP-PCN neurons. Furthermore, spatial segregation of VPd projections could play a role in the differential activation of certain subsets of DP-PCN neurons during the production of different displays.

\section{The mormyrid electromotor network as a CPG}

Research on the neural control of motor output has generally focused on model systems with easily quantified behaviors and readily accessible neurons. These advantages have led to concepts such as the CPG, a neural circuit that produces rhythmic output in the absence of external input (Marder and Bucher, 2001). The rhythmic activity of CPGs results from a combination of cellular and network properties, and many common features are found across a wide diversity of systems (Selverston, 1999; Marder and Bucher, 2001). Several observations from the current study and other recent findings on the mormyrid electromotor system suggest that it shares several of these properties (von der Emde et al., 2000; Carlson, 2002a), including recurrent inhibition, oscillatory activity in DP-PCN neurons, and bursting behavior in VPd neurons (Fig. 12).

In addition to driving EOD production, activity in $\mathrm{CN}$ leads to activation of the corollary discharge pathway, which ultimately leads to stereotyped bursts in VPd neurons that inhibit DP-PCN neurons. Such recurrent inhibition plays an important role in patterning rhythmic activity in several CPGs (Getting, 1989; Marder and Bucher, 2001). If DP-PCN neurons tend to fire tonically at rest, recurrent inhibition would provide a rate limiting input that would reset their activity, thereby inducing an oscilla- 
tory firing pattern that may be responsible for the resting electromotor rhythm (Friesen and Stent, 1978). External influences on these network components appear to disrupt this rhythm and induce the production of different electrical displays, which is similar to other CPGs in which modulatory inputs lead to changes in activity that result in different motor output patterns (Kupfermann and Weiss, 2001).

These commonalities between a vertebrate communication system and several well studied rhythmic motor systems suggest that many of the basic features involved in generating temporal patterns are shared across widely divergent networks with different functions. Compared with other vertebrate communication systems, the mormyrid electromotor network is relatively simple and the behavior is easily quantified, making it fairly straightforward to establish direct links between temporal patterning in signal production and neuronal control. It may therefore prove to be an excellent model system for exploring CPG function in vertebrate signal production.

\section{References}

Bell CC, Libouban S, Szabo T (1983) Pathways of the electric organ discharge command and its corollary discharges in mormyrid fish. J Comp Neurol 216:327-338.

Bell CC, Dunn K, Hall C, Caputi A (1995) Electric organ corollary discharge pathways in mormyrid fish: I. The mesencephalic command associated nucleus. J Comp Physiol [A] 177:449-462.

Bennett MVL, Pappas G, Aljure E, Nakajima Y (1967) Physiology and ultrastructure of electrotonic junctions. II. Spinal and medullary electromotor nuclei in mormyrid fish. J Neurophysiol 30:180-208.

Bullock TH, Bodznick DA, Northcutt RG (1983) The phylogenetic distribution of electroreception: evidence for convergent evolution of a primitive vertebrate sense modality. Brain Res Rev 6:25-46.

Carlson BA (2002a) Neuroanatomy of the mormyrid electromotor control system. J Comp Neurol 454:440-455.

Carlson BA (2002b) Electric signaling behavior and the mechanisms of electric organ discharge production in mormyrid fish. J Physiol (Paris) 96:405-419.

Carlson BA, Hopkins CD (2000) Neural mechanisms in the generation of electric signals in mormyrid fish. Soc Neurosci Abstr 26:1522.

Carlson BA, Hopkins CD (2001) Extracellular stimulation of descending electromotor nuclei in the mormyrid Brienomyrus brachyistis and the control of electric signaling behavior. In: Sixth International Congress of Neuroethology, p 221. Bonn, Germany: International Society for Neuroethology.

Carlson BA, Hopkins CD (2002) Two motor control centers for social communication displays in mormyrid electric fish. Soc Neurosci Abstr 28:37.

Doiron B, Chacron MJ, Maler L, Longtin A, Bastian J (2003) Inhibitory feedback required for oscillatory responses to communication but not prey stimuli. Nature 421:539-543.

Dowben R, Rose J (1953) A metal-filled microelectrode. Science 118:22-24.

Elekes K, Szabo T (1985) The mormyrid brainstem. III. Ultrastructure and synaptic organization of the medullary "pacemaker" nucleus. Neuroscience 15:431-443.

Faumont S, Simmers J, Meyrand P (1998) Activation of a lobster motor rhythm-generating network by disinhibition of permissive modulatory inputs. J Neurophysiol 80:2776-2780.

Friesen W, Stent G (1978) Neural circuits for generating rhythmic movements. Annu Rev Biophys Bioeng 7:37-61.

Gabbiani F, Koch C (1998) Principles of spike train analysis. In: Methods in Neuronal Modeling: from Ions to Networks, 2nd Edition (Koch C, Segev I, eds), pp 313-360. Cambridge, MA: MIT.
Getting PA (1989) Emerging principles governing the operation of neural networks. Annu Rev Neurosci 12:185-204.

Gomez L, Grant K, von der Emde G, Budelli R (1998) A model of the electromotor command system in Gnathonemus petersii. In: Fifth International Congress of Neuroethology, p 333. San Diego, CA: International Society for Neuroethology.

Grant K, Bell CC, Clausse S, Ravaille M (1986) Morphology and physiology of the brainstem nuclei controlling the electric organ discharge in mormyrid fish. J Comp Neurol 245:514-530.

Grant K, Von der Emde G, Sena LG, Mohr C (1999) Neural command of electromotor output in mormyrids. J Exp Biol 202:1399-1407.

Hopkins CD (1986) Behavior of Mormyridae. In: Electroreception (Bullock TH, Heiligenberg W, eds), pp 527-576. New York: Wiley.

Hopkins CD, Bass AH (1981) Temporal coding of species recognition signals in an electric fish. Science 212:85-87.

Kupfermann I, Weiss KR (2001) Motor program selection in simple model systems. Curr Opin Neurobiol 11:673-677.

Marder E, Bucher D (2001) Central pattern generators and the control of rhythmic movements. Curr Biol 11:R986-R996.

Metzner W (1999) Neural circuitry for communication and jamming avoidance in gymnotiform electric fish. J Exp Biol 202:1365-1375.

Moller P, Serrier J, Bowling D (1989) Electric organ discharge displays during social encounter in the weakly electric fish Brienomyrus niger L. (Mormyridae). Ethology 82:177-191.

Munemori J, Hara K, Kimura M, Sato R (1984) Statistical features of impulse trains in the cat's lateral geniculate neurons. Biol Cybern 50:167-172.

Noga B, Kettler J, Jordan L (1988) Locomotion produced in mesencephalic cats by injections of putative transmitter substances and antagonists into the medial reticular formation and the pontomedullary locomotor strip. J Neurosci 8:2074-2086.

Paulin MG (1992) Digital filters for firing rate estimation. Biol Cybern 66:525-531.

Pollack GS (2001) Analysis of temporal patterns of communication signals. Curr Opin Neurobiol 11:734-738.

Selverston A (1999) General principles of rhythmic motor pattern generation derived from invertebrate CPGs. Prog Brain Res 123:247-257.

Serrier J, Moller P (1989) Patterns of electric organ discharge activity in the weakly electric fish Brienomyrus niger L. (Mormyridae). Exp Biol 48:235-244.

Szabo T (1961) Anatomophysiologie de centres nerveux specifiques de quelques organes electriques. In: Bioelectrogenesis (Chagas C, del Carvahlo P, eds), pp 185-201. Amsterdam: Elsevier.

Szücs A (1998) Applications of the spike density function in analysis of neuronal firing patterns. J Neurosci Methods 81:159-167.

Teyssedre C, Boudinot M (1987) Rhythmicity as an intrinsic property of the mormyrids electromotor command system. Physiol Behav 41:201-207.

von der Emde G, Sena LG, Niso R, Grant K (2000) The midbrain precommand nucleus of the mormyrid electromotor network. J Neurosci 20:5483-5495.

Wang Y, Bieger D (1991) Role of solitarial GABAergic mechanisms in control of swallowing. Am J Physiol 261:R639-R646.

Wullimann MF, Northcutt RG (1990) Visual and electrosensory circuits of the diencephalon in mormyrids: an evolutionary perspective. J Comp Neurol 297:537-552. 